\title{
Information mining and visualization of highly cited papers on type-2 diabetes mellitus from ESI
}

\author{
Tong Zhai* and Lizhi Di
}

\begin{abstract}
A bibliometric analysis based on 1845 highly cited papers extracted from the Essential Science Indicators database was carried out to provide insights into publication performances and research characteristics of type-2 diabetes mellitus. Indicators were applied to evaluate the influence of the most productive journals, countries/territories, organizations and authors. Social network analysis was performed to evaluate and visualize the interaction among productive countries/territories, organizations, authors and keywords using VOSviewer software. Results obtained from this study can provide valuable information for researchers to better identify present and future hotspots in type-2 diabetes-related fields.
\end{abstract}

Keywords: Bibliometric methods, type-2 diabetes mellitus, highly cited papers, social network analysis.

TYPE-2 diabetes mellitus is the most common form of diabetes. It is a long-term metabolic disorder that is characterized by high blood sugar, insulin resistance and relative lack of insulin ${ }^{1,2}$. Type-2 diabetes as a chronic disease is associated with a 10-year shorter life expectancy. This is partly due to a number of complications with which it is associated, viz. 2-4 times the risk of cardiovascular disease (including ischaemic heart disease and stroke), a 20-fold increase in lower limb amputations and increased rates of hospitalizations. Now, the prevalence of type- 2 diabetes is increasing worldwide ${ }^{3-5}$. There are more researches involved with type-2 diabetes in order to improve human health. For example, Gerstein et al. ${ }^{6}$ studied the effects of intensive glucose lowering in type-2 diabetes. They found that the use of intensive therapy to target normal glycated haemoglobin levels for 3.5 years increased mortality and did not significantly reduce major cardiovascular events. Neal et al. $^{7}$ studied canagliflozin, and cardiovascular and renal events in type- 2 diabetes. Marso et $a l .{ }^{8}$ studied liraglutide and cardiovascular outcomes in type-2 diabetes.

The Essential Science Indicators (ESI) database (Clarivate Analytics) reveals emerging science trends as well as influential individuals, institutions, papers, journals and countries in a research field ${ }^{9-11}$. Article counts for ESI are derived from journals indexed in the Web of Science Core Collection (Science Citation Index Expanded (SCI) and Social Sciences Citation Index only) over a 10-year period. In ESI, papers are divided into 22 research fields

\footnotetext{
Tong Zhai is in the Tianjin University Library, Tianjin University, Tianjin 300072, PR China; Information Science Institute of Tianjin University, Tianjin University, Tianjin 300072, P.R. China; Lizhi Di is in the Tianjin Medical College, Tianjin 300222, PR China.

*For correspondence. (e-mail: zhaitong@tju.edu.cn)
}

and an article can be assigned to only one field. ESI highly cited paper (HCP) is one that belongs to the top $1 \%$ of papers in a research field published in a specified year. The HCPs help identify breakthrough research within a given field, and are used in the Web of Science (WoS) to identify and refine the most influential research papers. As for the field of type- 2 diabetes, according to our search, there are more than 130,000 papers in SCI Expanded. It is difficult for a researcher to read all these papers, especially for those new to the field. Bibliometric technique is an efficient method for analysing the papers in a research field ${ }^{12-14}$. In this study, we employ the bibliometric method to analyse the ESI HCPs on type2 diabetes. The characteristics and relationship networks of the HCPs are also presented.

\section{Methodology}

In this study, the publication data were obtained from the on-line version of Web of Science Core Collection database. All papers on type-2 diabetes were searched on the basis of topic (title, abstract, author keywords, keywords plus). As mentioned earlier, there were more than 130,000 papers on type-2 diabetes from 2007 to 2017 in SCI Expanded. Among these, 1845 papers were marked as ESI HCPs. The data of total 1845 HCPs were exported from the Web of Science Core Collection and analysed using VOSviewer, a software tool for constructing and visualizing bibliometric networks ${ }^{15-20}$.

\section{Results and discussion}

Figure 1 shows the number of HCPs published each year. In 2007, there were $136 \mathrm{HCPs}$ on type-2 diabetes. In 
2008 , the number was reduced to 126 . In the following six years, the number of HCPs increased steadily, and in 2014, it reached the highest value. The number of HCPs in 2015 and 2016 decreased slightly. Due to the hysteretic nature of the database, data in 2017 are not very representative. The 1845 HCPs on type- 2 diabetes are distributed in 17 ESI research fields. Most of the papers are assigned to the 'clinical medicine' field, which accounts for $65 \%$ of the total. The next five research fields include biology and biochemistry (9.4\%), agricultural sciences $(6.2 \%)$, pharmacology and toxicology (5\%), social sciences, general (4.7\%), and molecular biology and genetics (4.6\%) (Figure 2).

Open-access status is provided across the WoS platform. There are degrees of open access: OA (gold), OA (green published) and OA (green accepted). OA (gold) is identified as fully published articles available from the publisher without charge. OA (green published) is final published articles available without charge from a repository. OA (green accepted) is peer-reviewed accepted manuscripts available without charge from a repository. OA articles are available to anyone with a computer and an internet connection. This makes them have a broad audience, which helps improve their impact. For the 1845 HCPs, $66.4 \%$ is OA articles, specifically as

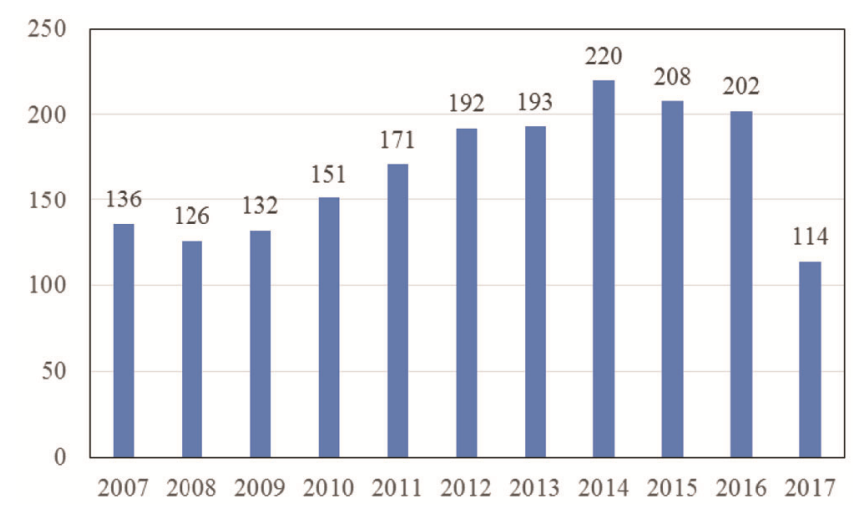

Figure 1. The number of highly cited papers published each year.

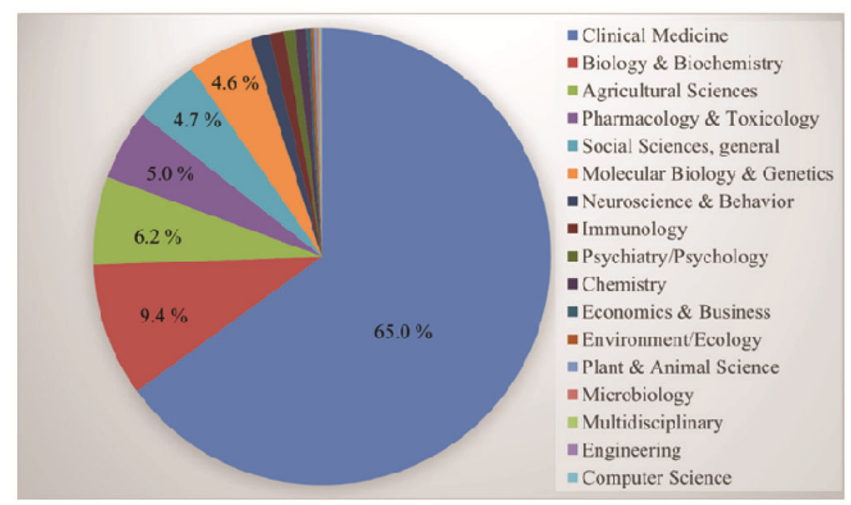

Figure 2. Distribution of 1845 highly cited papers in ESI research fields. follows: OA (gold) - 55.9\%, OA (green accepted) - 9.2\% and OA (green published) $-1.3 \%$ (Figure 3 ). There are 151 OA (gold) HCPs published by Diabetes Care, 22 OA (green accepted) HCPs by Nature and 4 OA (green published) HCPs published by the Journal of Clinical Investigation.

The HCPs on type-2 diabetes were published in 413 journals. Table 1 shows the number of HCPs and their citation indicators in the top 15 productive journals. Diabetes Care is the most productive journal which published 158 HCPs. The number of HCPs (PN) published by Lancet and New England Journal of Medicine ranks second $(\mathrm{PN}=71)$ and third $(\mathrm{PN}=67)$ in the top 15 productive journals respectively. For the top 15 journals, HCPs published by New England Journal of Medicine have the highest total citations (TC). They have been cited 43,217 times in total. However, HCPs published by Circulation have the highest average citations (AC), which reached 691 times. Figure 4 shows AC values and impact factor (IF) of each of the top 15 productive journals. For HCPs, variation trend of AC values is not consistent with that of journal IF. The most obvious one is Circulation. The IF of this journal ranks only sixth in the top 15 journals, however, its $\mathrm{AC}$ value ranks first.

The 1845 HCPs papers on type-2 diabetes are distributed in 82 countries/territories around the world. Among the 1845 selected publications, 855 papers (46.3\% of the total studied papers) were published with international collaboration. Table 2 shows the top 15 most productive countries. They include 11 European countries/territories, two American countries, one Oceania country and one Asian country/territory, which are dominated by developed countries/territories, demonstrating the positive role of economic capabilities in promoting academic levels. USA is the most productive country with 1026 HCPs $(55.6 \%$ of the total studied papers). TC of USA HCPs is also the highest in the top

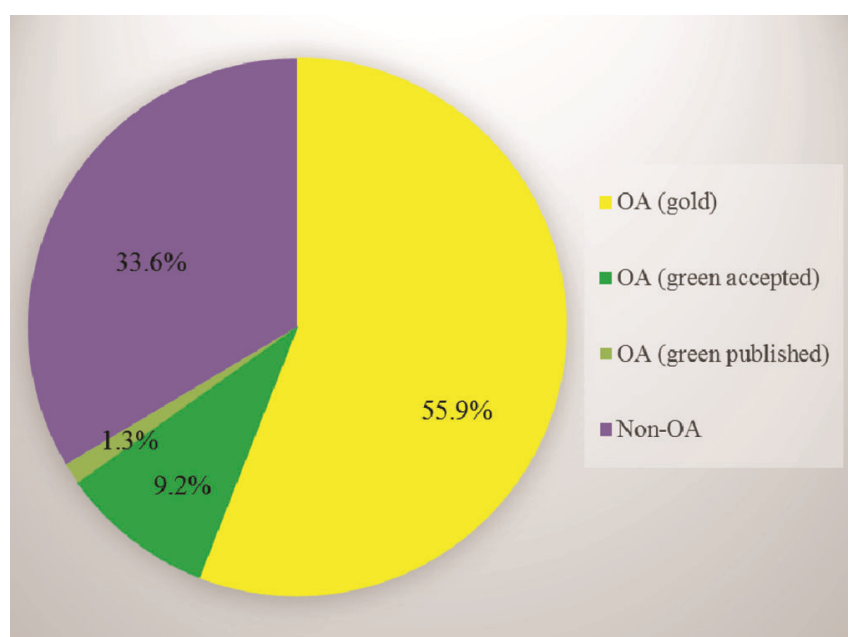

Figure 3. Open access status of the 1845 highly cited papers.

CURRENT SCIENCE, VOL. 116, NO. 12, 25 JUNE 2019 
GENERAL ARTICLES

Table 1. The numbers of highly cited papers (HCPs) and their citation indicators in top 15 productive journals

\begin{tabular}{|c|c|c|c|}
\hline Journal & PN & $\begin{array}{l}\text { Total citations } \\
\text { (TC) }\end{array}$ & $\begin{array}{c}\text { Average citations } \\
\text { (AC) }\end{array}$ \\
\hline Diabetes Care & 158 & 31,099 & 196.8 \\
\hline Lancet & 71 & 26,052 & 366.9 \\
\hline New England Journal of Medicine & 67 & 43,217 & 645.0 \\
\hline JAMA Journal of the American Medical Association & 59 & 17,736 & 300.6 \\
\hline Diabetes & 55 & 12,091 & 219.8 \\
\hline Diabetologia & 51 & 9,622 & 188.7 \\
\hline Circulation & 44 & 30,403 & 691.0 \\
\hline Diabetes Obesity Metabolism & 41 & 5,238 & 127.8 \\
\hline Nature & 41 & 21,731 & 530.0 \\
\hline Lancet Diabetes Endocrinology & 33 & 2,466 & 74.7 \\
\hline Nature Reviews Endocrinology & 33 & 4,237 & 128.4 \\
\hline Journal of Clinical Endocrinology Metabolism & 33 & 6,844 & 207.4 \\
\hline Annals of Internal Medicine & 32 & 5,617 & 175.5 \\
\hline Journal of Clinical Investigation & 30 & 7,473 & 249.1 \\
\hline Journal of the American College of Cardiology & 22 & 6,259 & 284.5 \\
\hline
\end{tabular}

PN, Number of highly cited papers.

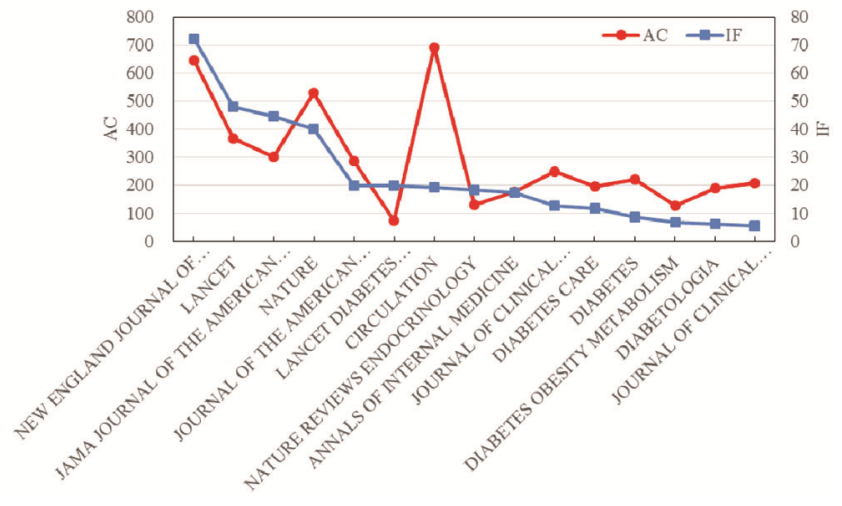

Figure 4. Highly cited papers average citations (HCP AC) values and impact factor of each of the top 15 productive journals.

15 countries/territories. For $\mathrm{AC}$, Scotland $(\mathrm{AC}=378.0)$ ranks first in the top 15 countries/territories. The HCP average growth rate per annum (AGRA) from 2007 to 2016 was calculated. The Agra of The Netherlands is the highest in the top 15 countries/territories and Switzerland experienced negative growth during 2007-2016. The Peoples' R China was the only developing country in the top 15 countries with $77 \mathrm{HCPs}$ and ranked 15 th. It presented a strong growth momentum during the past decade, with AGRA reaching 48.1\%.

International collaborations of the top 15 countries/ territories were studied (Table 2). The internationally collaborative papers of USA accounted for $54.3 \%$ of all HCPs in the country, which is the least (ICPC (the percentage of international collaboration publications in a country/territory) indicator) in the top 15 countries/ territories. However, the internationally collaborative papers of USA accounted for $30.2 \%$ of all HCPs in the world, which is the highest (ICPW indicator) in the top 15 countries/territories. The ratio of collaborative to noncollaborative papers of USA is moderate. For the other 14 countries/territories, the ICPC values are very high $(>77 \%)$. On the one hand, it shows that the 14 countries/ territories have a high openness. On the other, it suggests that HCPs of these 14 countries/territories depend highly on other countries/territories, and that independent research ability is weak. Links is an indicator (VOSviewer) which is used to represent the number of connections of one factor (e.g. one country/territory) with other factors (e.g. the other 14 countries/territories). The links of all the 15 top countries/territories are 14, which indicates that all of the top 15 countries/territories collaborated with each other. Total link strength (TLS) is another indicator (VOSviewer), which is used to represent collaboration intensity of the selective factors (countries/ territories). USA has the highest collaboration intensity $(\mathrm{TLS}=1126)$ in the top 15 countries/territories. Figure 5 shows visualization of international collaborations and top 10 link strength combinations between the top 15 countries/territories. In network visualization, size of the circle of an item is determined by the weight of that item. The higher the weight of an item, larger is the circle of that item. The link strength between USA and England is maximum, with the number of USA-England collaboration HCPs being 163. Among the top 10 link strength combinations, the number of such combinations involving USA is 8. In the collaborations, USA is at the central position, which shows its research potential in the type-2 diabetes field. The colours in Figure 5 are used to distinguish between different clusters (a cluster is a set of closely related nodes). The clustering technique used by VOSviewer is discussed by Waltman and coworkers $^{21,22}$.

The first author of a paper is usually the person who has undertaken a major part of the research work. The corresponding author (CA) of a paper is the person to whom readers can address questions, request for materials, or even provide suggestions for further work, and is usually 
Table 2. Top 15 most productive countries and related indicators

\begin{tabular}{|c|c|c|c|c|c|c|c|c|}
\hline Country/territory & $\mathrm{PN}$ & $\mathrm{TC}$ & $\mathrm{AC}$ & $\begin{array}{c}\text { AGRA } \\
(\%)\end{array}$ & $\begin{array}{c}\text { ICPC } \\
(\%)\end{array}$ & $\begin{array}{c}\text { ICPW } \\
(\%)\end{array}$ & TLS & Links \\
\hline USA & 1026 & 263,247 & 256.6 & 9.9 & 54.3 & 30.2 & 1126 & 14 \\
\hline England & 378 & 97,135 & 257.0 & 30.1 & 78.3 & 16.0 & 868 & 14 \\
\hline Germany & 220 & 49,092 & 223.1 & 42.4 & 83.6 & 10.0 & 655 & 14 \\
\hline Canada & 195 & 63,625 & 326.3 & 19.2 & 77.9 & 8.2 & 473 & 14 \\
\hline Italy & 190 & 51,952 & 273.4 & 33.9 & 86.8 & 8.9 & 618 & 14 \\
\hline Australia & 173 & 48,755 & 281.8 & 34.7 & 82.1 & 7.7 & 473 & 14 \\
\hline The Netherlands & 152 & 38,370 & 252.4 & 51.8 & 81.6 & 6.7 & 525 & 14 \\
\hline Denmark & 151 & 43,703 & 289.4 & 26.0 & 80.1 & 6.6 & 483 & 14 \\
\hline France & 150 & 48,858 & 325.7 & 21.1 & 88.0 & 7.2 & 530 & 14 \\
\hline Sweden & 125 & 33,944 & 271.6 & 31.7 & 90.4 & 6.1 & 445 & 14 \\
\hline Switzerland & 110 & 28,418 & 258.3 & -5.0 & 88.2 & 5.3 & 320 & 14 \\
\hline Spain & 89 & 21,349 & 239.9 & 58.7 & 82.0 & 4.0 & 343 & 14 \\
\hline Finland & 88 & 28,576 & 324.7 & 0.0 & 87.5 & 4.2 & 329 & 14 \\
\hline Scotland & 87 & 32,887 & 378.0 & 18.6 & 80.5 & 3.8 & 334 & 14 \\
\hline Peoples' R China & 77 & 17,262 & 224.2 & 48.1 & 79.2 & 3.3 & 174 & 14 \\
\hline
\end{tabular}

TC, Total citations; AC, Average citations; AGRA, Average growth rate per annum (2007-16); ICPC, Percentage of international collaboration publications in a country/territory; ICPW, Percentage of international collaboration publications in the world; TLS, Total link strength.

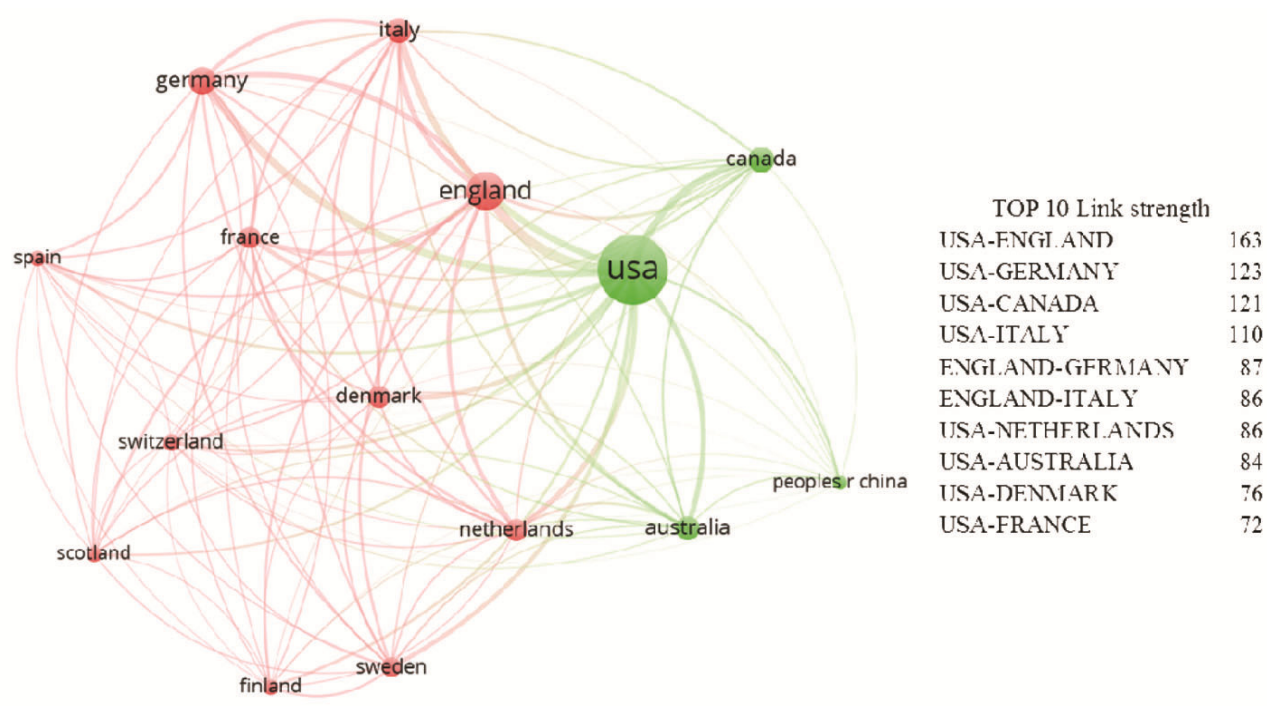

Figure 5. Visualization of international collaborations and top 10 link strength combinations between the top 15 countries/territories.
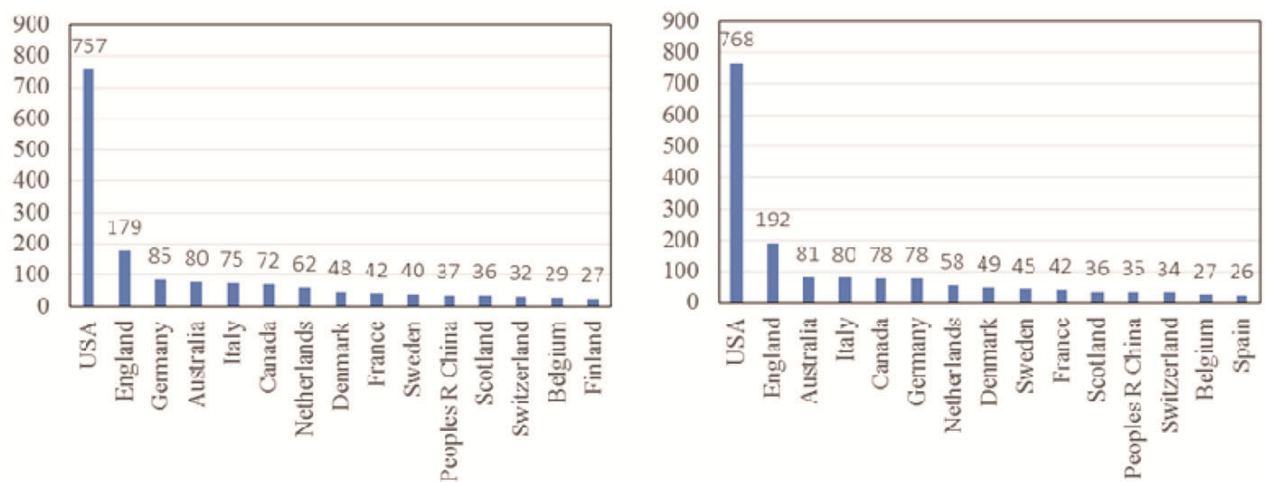

Figure 6. Top 15 productive countries/territories based on $(\boldsymbol{a})$ first authored and $(\boldsymbol{b})$ corresponding authored HCPs respectively. 
Table 3. Top 16 most productive corresponding authors

\begin{tabular}{lrlr}
\hline Corresponding author (CA) & PN & Organization/institution & TC \\
\hline F. B. Hu & 16 & Harvard Univ & 3271 \\
G. I. Shulman & 13 & Yale Univ & 3104 \\
R. A. DeFronzo & 12 & Univ Texas Hlth Sci Ctr San Antonio & 3043 \\
D. J. Drucker & 11 & Univ Toronto & 4166 \\
M. A. Nauck & 10 & Ruhr Univ Bochum; Diabet Zentrum Bad Lauterberg & 1871 \\
E. Ferrannini & 9 & Univ Pisa; CNR Inst Clin Physiol & 1187 \\
J. Rosenstock & 8 & Med City Dallas Hosp, Dallas Diabet and Endocrine Ctr & 1093 \\
J. B. Buse & 7 & Univ N Carolina & 1714 \\
P. D. Cani & 7 & Catholic Univ Louvain & 1397 \\
D. G. Hardie & 7 & Univ Dundee & 2010 \\
G. Targher & 7 & Univ Verona & 2107 \\
P. C. Butler & 6 & Univ Calif Los Angeles & 1417 \\
R. R. Holman & 6 & Churchill Hosp; Univ Oxford \\
B. Neal & 6 & Univ Sydney; Royal Prince Alfred Hosp & 4942 \\
J. M. Olefsky & 6 & Univ Calif San Diego & 375 \\
Z. M. Younossi & 6 & Inova Fairfax Hosp; Inova Hlth Syst, Betty and & 2831 \\
& & Guy Beatty Ctr Integrated Res & 1851 \\
\hline
\end{tabular}

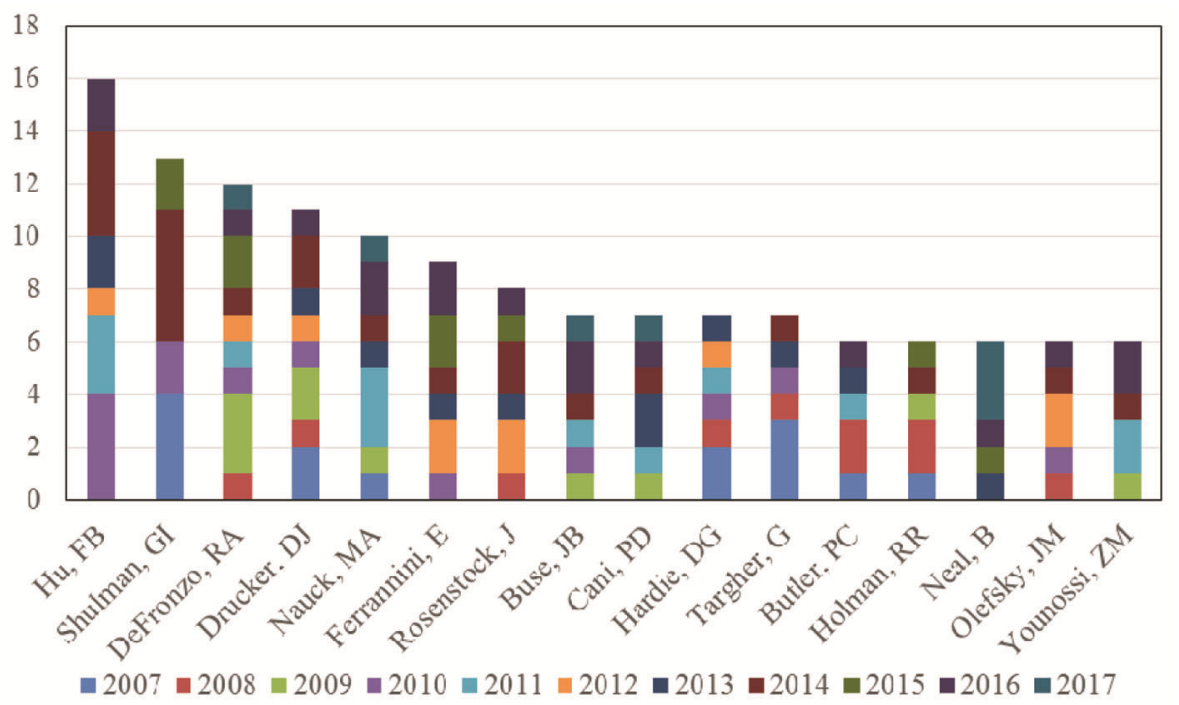

Figure 7. Time distribution of the top 16 most productive corresponding authors' HCPs.

the director of a research project. The distribution of the 1845 HCPs in the world based on first author and corresponding author was studied. As shown in Figure 6, the number of first-authored HCPs by the top 15 countries/territories is similar to that of correspondingauthored HCPs. USA and England are first and second productive countries/territories, based on both first author and corresponding author. USA has an obvious advantage compared with other countries/territories.

Table 3 shows the top 16 most productive corresponding authors. They come from different organizations and as corresponding authors have published 137 HCPs in total. F. B. Hu (Harvard University, USA) is the most productive corresponding author. As corresponding author, he has published $16 \mathrm{HCPs}$ with a total citation of 3271. As shown in Figure 7, these publications were distributed as follows: 2010-four HCPs, 2011 - three
HCPs, 2012 - one HCP, 2013 - two HCPs, 2014 - four HCPs and 2016 - two HCPs. Hu has conducted detailed analyses of many dietary and lifestyle factors and risks of diabetes, including sugar-sweetened beverages, coffee, red meat, saturated and polyunsaturated fatty acids, iron and dietary patterns. These findings have contributed to current public health recommendations and policies for prevention of chronic diseases. For example, an important research finding of $\mathrm{Hu}$ is that higher consumption of sugar-sweetened beverages is associated with the development of metabolic syndrome and type- 2 diabetes. Research data provide empirical evidence that intake of sugar-sweetened beverages should be limited to reduce obesity-related risk of chronic metabolic diseases ${ }^{23}$.

R. R. Holman (Churchill Hospital and University of Oxford, UK) has the highest total citations $(\mathrm{TC}=4942)$ among the top 16 corresponding authors. The six HCPs of 
Table 4. Top 15 most productive organizations and related indicators

\begin{tabular}{|c|c|c|c|c|c|}
\hline Organizations/institution & PN & $\mathrm{TC}$ & $\mathrm{AC}$ & TLS & Links \\
\hline Harvard Univ (USA) & 197 & 61,880 & 314.1 & 250 & 14 \\
\hline Brigham Womens HOSP (USA) & 81 & 22,864 & 282.3 & 173 & 14 \\
\hline Univ Washington (USA) & 75 & 25,380 & 338.4 & 142 & 14 \\
\hline Univ Oxford (England) & 70 & 29,851 & 426.4 & 176 & 13 \\
\hline Univ N Carolina (USA) & 70 & 32,839 & 469.1 & 163 & 14 \\
\hline Univ Copenhagen (Denmark) & 67 & 17,310 & 258.4 & 77 & 14 \\
\hline Univ Toronto (Canada) & 64 & 19,951 & 311.7 & 69 & 13 \\
\hline Univ Michigan (USA) & 54 & 19,433 & 359.9 & 146 & 13 \\
\hline Univ Sydney (Australia) & 54 & 15,446 & 286.0 & 32 & 10 \\
\hline Univ London Imperial Coll Sci Technol MED (England) & 52 & 15,453 & 297.2 & 148 & 13 \\
\hline Yale Univ (USA) & 50 & 17,832 & 356.6 & 42 & 11 \\
\hline Univ Helsinki (Finland) & 48 & 17,115 & 356.6 & 121 & 13 \\
\hline Univ Cambridge (England) & 47 & 14,237 & 302.9 & 122 & 12 \\
\hline Massachusetts Gen Hosp (USA) & 46 & 20,436 & 444.3 & 167 & 14 \\
\hline Columbia Univ (USA) & 45 & 18,877 & 419.5 & 36 & 10 \\
\hline
\end{tabular}

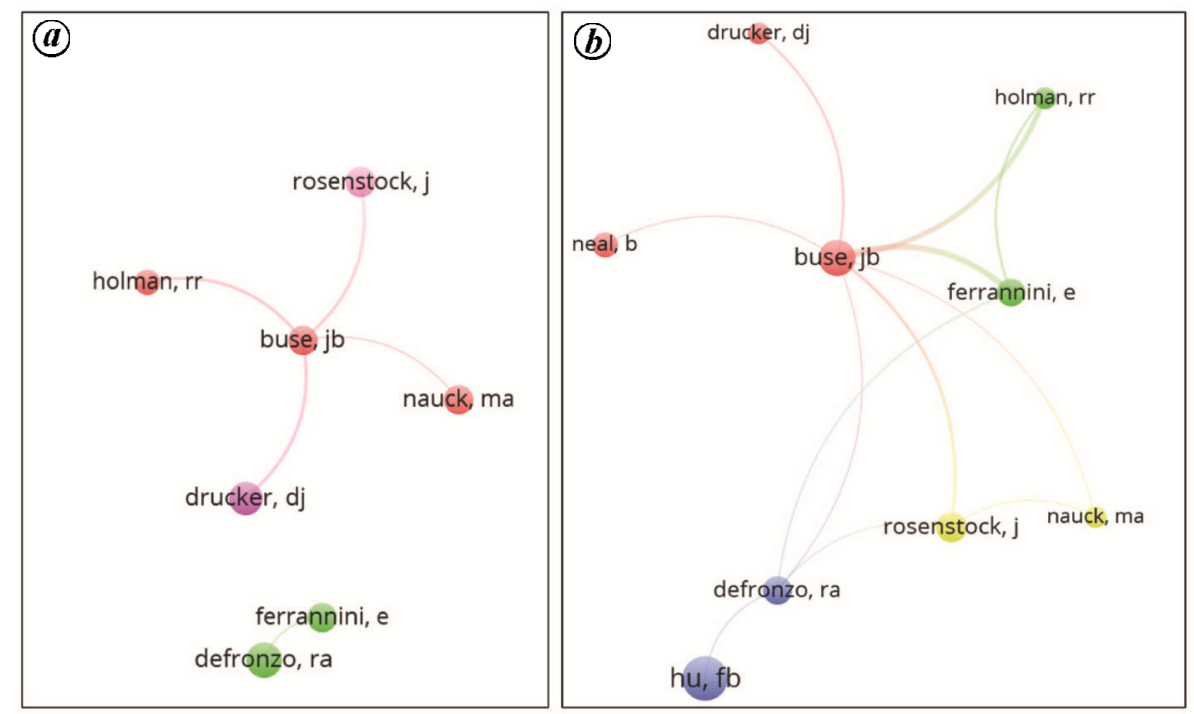

Figure 8. Collaborative relationships between the top 16 corresponding authors in (a) 137 HCPs and (b) total 1845 HCPs.

Holman were distributed as follows: 2007 - one HCP, 2008 - two HCPs, 2009 - one HCP, 2014 - one HCP and 2015 - one HCP. B. Neal has the least total citations $(\mathrm{TC}=375)$. One of the main reasons is because the HCPs of Neal were published in recent years: 2013 - one HCP, 2015 - one HCP, 2016 - one HCP and 2017 - two HCPs. Holman directs the 10-year UK Prospective Diabetes Study post-trial monitoring programme and operates the 20-year UK Prospective Diabetes Study. He has designed and run many multicentre studies that focus primarily on the prevention, appropriate treatment and cardiovascular risk reduction in people with type- 2 diabetes. For example, his research showed that a continued reduction in microvascular risk and emergent risk reductions for myocardial infarction and death from any cause were observed during 10 years of post-trial follow-up. A continued benefit after metformin therapy was evident among overweight patients ${ }^{24}$.
Figure $8 a$ shows the collaborative relationships between the top 16 corresponding authors for the 137 HCPs. J. B. Buse has collaborative relationships with R. R. Holman, J. Rosenstock, M. A. Nauck and D. J. Drucker, E. Ferrannini has collaborative relationship with R. A. DeFronzo. There is no collaborative relationship between other corresponding authors in the 137 HCPs. Figure $8 b$ shows the collaborative relationship between the top 16 corresponding authors in total 1845 HCPs. Again J. B. Buse has the most collaborators: R. R. Holman, J. Rosenstock, M. A. Nauck, D. J. Drucker, E. Ferrannini, R. A. DeFronzo and B. Neal. R. A. DeFronzo has four collaborators: E. Ferrannini, F. B. Hu, J. Rosenstock and J. B. Buse. J. Rosenstock and E. Ferrannini both have three collaborators. J. B. Buse is an author with high openness. R. R. Holman and E. Ferrannini are the top two authors who have collaborated closely with J. B. Buse based on total 1845 HCPs. 
GENERAL ARTICLES

Table 5. List of author keywords (occurrences $\geq 10$ ) and their total link strength

\begin{tabular}{|c|c|c|c|c|c|}
\hline Author keywords & Occurrences & $\begin{array}{l}\text { Total link } \\
\text { strength }\end{array}$ & Author keywords & Occurrences & $\begin{array}{c}\text { Total link } \\
\text { strength }\end{array}$ \\
\hline Obesity & 120 & 256 & Diet & 15 & 35 \\
\hline Type-2 diabetes & 133 & 189 & Nutrition & 17 & 35 \\
\hline Diabetes & 107 & 153 & Cholesterol & 16 & 34 \\
\hline Metabolic syndrome & 69 & 151 & Adipose tissue & 15 & 33 \\
\hline Insulin resistance & 71 & 147 & Dyslipidemia & 15 & 33 \\
\hline Cardiovascular disease & 57 & 129 & Exercise & 16 & 33 \\
\hline Inflammation & 65 & 121 & Metformin & 22 & 33 \\
\hline Meta-analysis & 55 & 95 & Oxidative stress & 20 & 31 \\
\hline Diabetes mellitus & 62 & 92 & Prevention & 12 & 28 \\
\hline Epidemiology & 38 & 82 & Glucose & 11 & 27 \\
\hline Cancer & 26 & 59 & Blood pressure & 14 & 26 \\
\hline Hypertension & 28 & 57 & Vitamin D & 12 & 26 \\
\hline Type-2 diabetes mellitus & 46 & 54 & Non-alcoholic fatty liver disease & 11 & 25 \\
\hline Insulin & 24 & 50 & Triglycerides & 11 & 25 \\
\hline Review & 26 & 50 & Heart failure & 14 & 24 \\
\hline Risk factors & 19 & 45 & Sglt2 inhibitor & 18 & 24 \\
\hline Cardiovascular diseases & 23 & 43 & Stroke & 11 & 24 \\
\hline AHA scientific statements & 14 & 40 & Nafld & 12 & 23 \\
\hline Lipids & 13 & 37 & Cardiovascular risk & 12 & 22 \\
\hline Metabolism & 19 & 37 & Aging & 14 & 20 \\
\hline Mortality & 20 & 37 & Insulin sensitivity & 13 & 19 \\
\hline Systematic review & 24 & 37 & Alzheimer's disease & 11 & 18 \\
\hline Atherosclerosis & 18 & 35 & Physical activity & 12 & 18 \\
\hline
\end{tabular}

brigham \& womens hosp

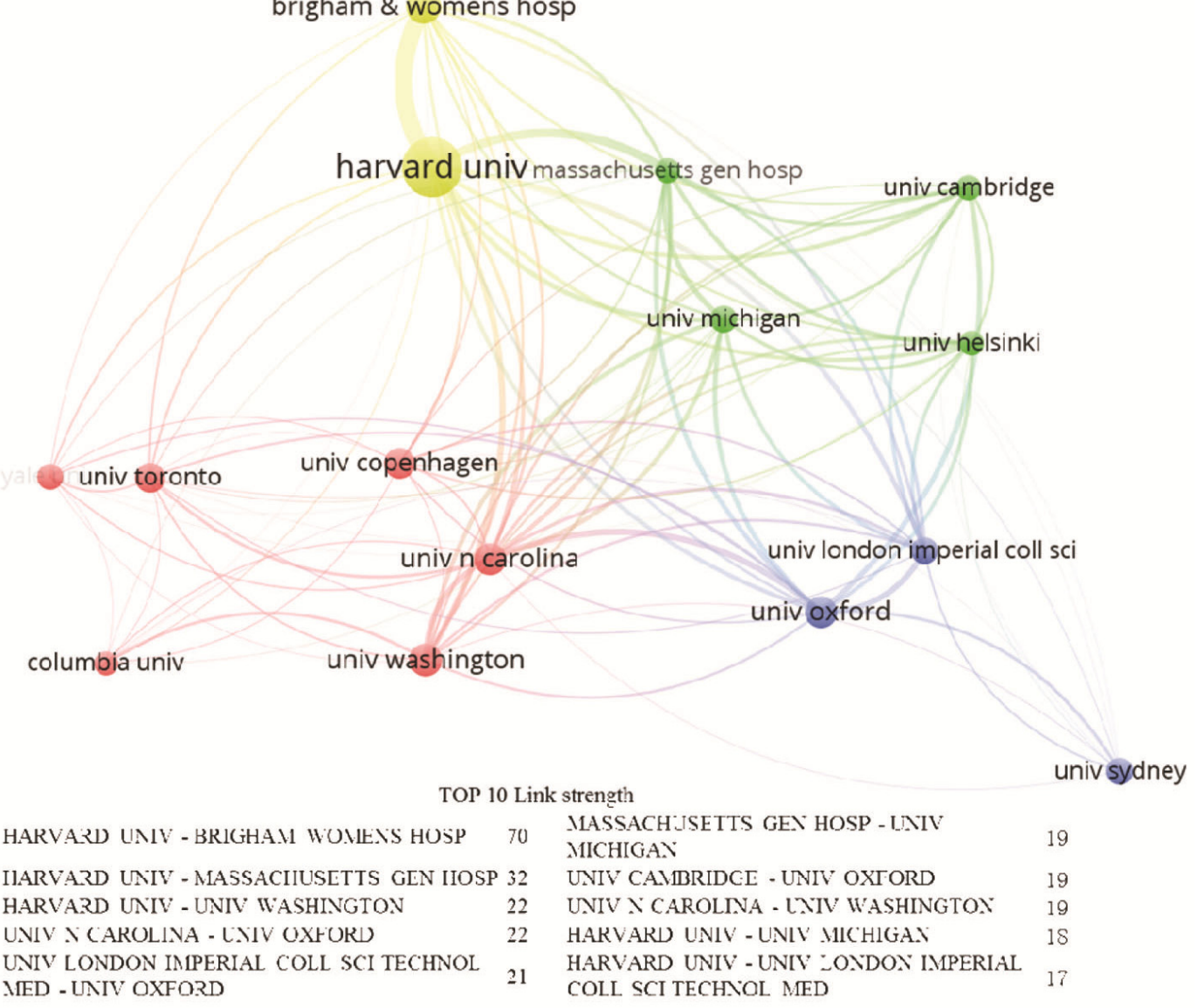

Figure 9. Visualization of collaborations and top 10 link strength combinations between the top-15 organizations. 


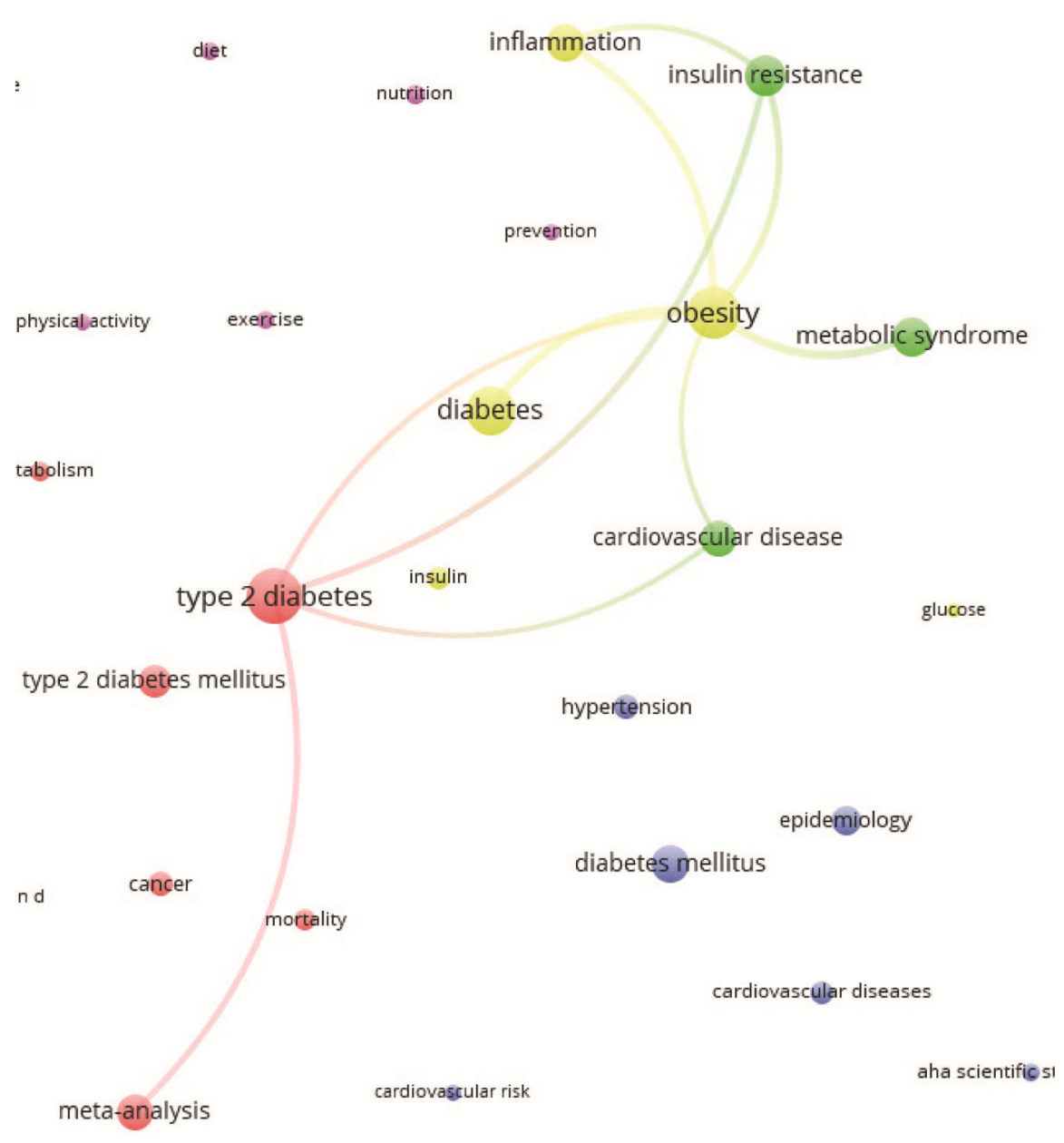

Figure 10. Top 10 closely connected groups of author keywords.

At the institution level, the 1845 HCPs on type-2 diabetes were from 3483 organizations around the world. Table 4 illustrates the primary performance of the top 15 most productive organizations. Among them, eight organizations are in USA, three in England, and one each in Denmark, Canada, Australia and Finland. Harvard University takes the leading position not only in total publications $(\mathrm{PN}=197)$, but also in total citations $(\mathrm{TC}=61880)$ and collaboration intensity $(\mathrm{TLS}=250)$. As for average citations, University of North Carolina $(\mathrm{AC}=469.1)$ ranks first in the top 15 organizations. The links of six organizations (Harvard University, Brigham and Women's Hospital, University Washington, University of North Carolina, University Copenhagen, Massachusetts General Hospital) are 14, which indicates that each of them collaborated with other 14 organizations. Figure 9 shows the visualization of collaborations and top 10 link strength combinations between the top 15 organizations. The link strength between Harvard University and Brigham and Women's Hospital is maximum, with number of collaborative HCPs being 70. The link strength between Harvard University and Massachusetts General
Hospital ranks second. Brigham and Women's Hospital and Massachusetts General Hospital are both teaching hospitals of the Harvard Medical School. This could be the reason they collaborated closely with Harvard University. The colours in Figure 9 are used to distinguish between different clusters.

Table 5 shows the author keywords (occurrences $\geq 10$ ) and their total link strength. Among the author keywords, 'diabetes' related words appeared the most, including 'type-2 diabetes', 'diabetes', 'diabetes mellitus' and 'type-2 diabetes mellitus'. 'Obesity' is the second-most common word that appears. In particular, it has the highest total link strength, which illustrates 'diabetes' as the keyword which is most closely connected with other keywords. The occurrences of 'insulin' related keywords (including 'insulin resistance', 'insulin' and 'insulin sensitivity') are also obvious. As can be seen from the listed author keywords, type 2 diabetes is associated with many other diseases, such as 'cardiovascular disease', 'inflammation', 'cancer', 'hypertension', 'atherosclerosis', 'dyslipidemia', 'non-alcoholic fatty liver disease', 'heart failure', 'stroke', 'nafld' and 'Alzheimer's disease'. 

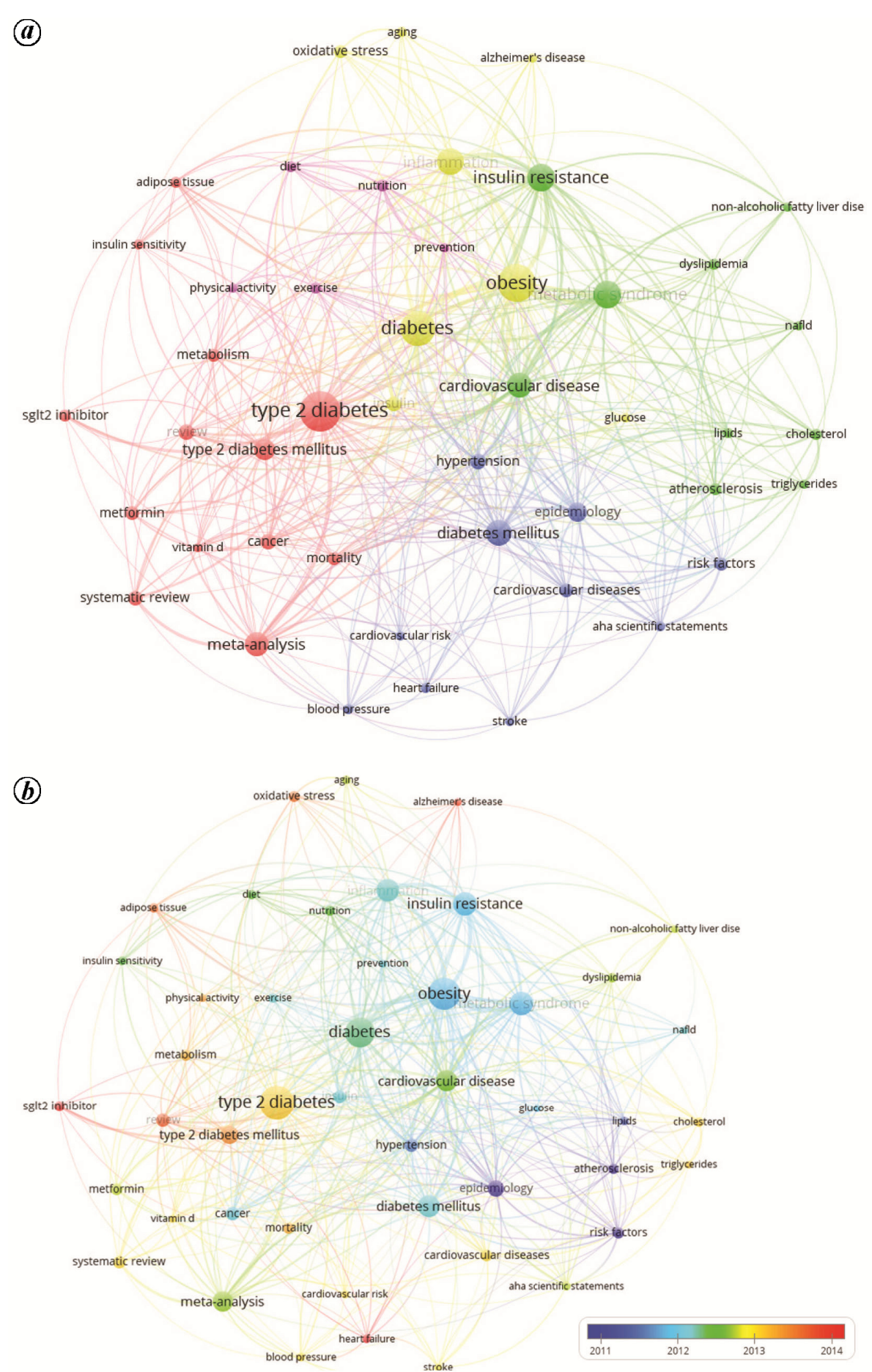

Figure 11. (a) network visualization and (b) overlay visualization of author keywords (occurrences $\geq 10$ ).

Figure 10 shows the top 10 closely connected groups of author keywords. The link strength between 'obesity' and 'metabolic syndrome' is maximum, which indicates that they appeared at the same time in 31 HCPs. The second is the group of 'obesity' and 'diabetes' (link strength $=28$ ), and the third is the group of 'obesity' and 'inflammation' (link strength $=24$ ).

Figure $11 a$ shows the network visualization of author keywords (occurrences $\geq 10$ ). The entire network of the
46 author keywords (occurrences $\geq 10$ ) is presented. The 46 author keywords have been divided into five groups and each group presented with a certain colour. In network visualization, the colour of author keywords is determined by the cluster to which the item belongs, according to VOSviewer. Figure $11 b$ shows the overlay visualization of author keywords (occurrences $\geq 10$ ). The author keywords in overlay visualization are coloured differently. The colour of an author keyword is determined by 
the publication year (or average publication year) of the item. For example, for the $1845 \mathrm{HCPs}$, 'sglt2 inhibitor' and 'heart failure' occur frequently in recent years. However, 'epidemiology' and 'atherosclerosis' have occurred frequently in previous years.

\section{Conclusion}

In this study, we have employed the bibliometric method to analyse HCPs on type-2 diabetes mellitus from ESI. The influence of most productive journals, countries/ territories, organizations and authors was evaluated. As for countries/territories, USA is the most productive country and has the highest total citations; Scotland has the highest average citations; The Netherlands has the highest AGRA values. At the institution level, Harvard University is the most productive organization and has the highest total citations; University of North Carolina has the highest average citations. F. B. Hu, (Harvard University) is the most productive corresponding author and R. R. Holman who ('Churchill Hospital' and 'University of Oxford') has the highest total citations. The interaction among productive countries/territories, organizations, authors and keywords was analysed. In international collaborations, USA is at the central position, which shows its research potential in type-2 diabetes mellitus field. For organizations, Harvard University has the highest collaboration intensity. For corresponding authors, J. B. Buse has the most collaborators. The relationship networks among productive countries/territories, organizations, authors and keywords were also visualized using VOSviewer.

1. Lei, X. Y. and Huang, S., Enrichment of minor allele of SNPs and genetic prediction of type-2 diabetes risk in British population. PLOS ONE, 2017, 12, 1-13.

2. Du, Z. H. and Uversky, V. N., A comprehensive survey of the roles of highly disordered proteins in type-2 diabetes. Intern. $J$. Mol. Sci., 2017, 18, 1-44.

3. Compston, J., Type 2 diabetes mellitus and bone. J. Intern. Med., 2018, 283, 140-153.

4. Zheng, Y., Ley, S. H. and Hu, F. B., Global aetiology and epidemiology of type-2 diabetes mellitus and its complications. Nature Rev. Endocrinol., 2018, 14, 88-98.

5. Lascar, N., Brown, J., Pattison, H., Barnett, A. H., Bailey, C. J. and Bellary, S., Type 2 diabetes in adolescents and young adults. Lancet Diabetes Endocrinol., 2018, 6, 69-80.

6. Gerstein, H. C. et al., Effects of intensive glucose lowering in type-2 diabetes. N. Engl. J. Med., 2008, 358, 2545-2559.

7. Neal, B. et al., Canagliflozin and cardiovascular and renal events in type-2 diabetes. N. Engl. J. Med., 2017, 377, 644-657.

8. Marso, S. P. et al., Liraglutide and cardiovascular outcomes in type-2 diabetes. N. Engl. J. Med., 2016, 375, 311-322.

9. Bornmann, L., Bauer, J. and Schlagberger, E. M., Characteristics of highly cited researchers 2015 in Germany. Scientometrics, 2017, 111, 543-545.
10. Harzing, A. W., Health warning: might contain multiple personalities - the problem of homonyms in Thomson Reuters Essential Science Indicators. Scientometrics, 2015, 105, 2259-2270.

11. Cova, T., Pais, A. and Formosinho, S. J., Iberian universities: a characterisation from ESI rankings. Scientometrics, 2013, 94, 1239-1251.

12. Valderrama, P., Escabias, M., Jimenez-Contreras, E., RodriguezArchilla, A. and Valderrama, M. J., Proposal of a stochastic model to determine the bibliometric variables influencing the quality of a journal: application to the field of dentistry. Scientometrics, 2018, 115, 1087-1095

13. Li, W. J., Dong, H., Yu, H., Wang, D. and Yu, H. B., Global characteristics and trends of research on ceramic membranes from 1998 to 2016: based on bibliometric analysis combined with information visualization analysis. Ceram. Int., 2018, 44, 69266934.

14. Merigo, J. M., Pedrycz, W., Weber, R. and de la Sotta, C., Fifty years of information sciences: a bibliometric overview. Inf. Sci., 2018, 432, 245-268.

15. Yeung, A. W. K., Tzvetkov, N. T. and Atanasov, A. G., When neuroscience meets pharmacology: a neuropharmacology literature analysis. Front. Neurosci., 2018, 12, 1-7.

16. Park, J. Y. and Nagy, Z., Comprehensive analysis of the relationship between thermal comfort and building control research-a data-driven literature review. Renew. Sustain. Energ. Rev., 2018, 82, 2664-2679.

17. Farzanegan, R. et al., An overview of tracheal stenosis research trends and hot topics. Arch. Iran. Med., 2017, 20, 598-607.

18. Peykari, N. et al., Scientometric study on non-communicable diseases in Iran: a review article. Iran. J. Public Health, 2018, 47, 936-943.

19. Yeung, A. W. K., Heinrich, M. and Atanasov, A. G., Ethnopharmacology - a bibliometric analysis of a field of research meandering between medicine and food science? Front. Pharmacol., 2018, 9, 1-15.

20. Yeung, A. W. K., Bibliometric study on functional magnetic resonance imaging literature (1995-2017) concerning chemosensory perception. Chemosens. Percept., 2018, 11, 42-50.

21. Waltman, L., van Eck, N. J. and Noyons, E. C. M., A unified approach to mapping and clustering of bibliometric networks. J. Informetr., 2010, 4, 629-635.

22. Waltman, L. and van Eck, N. J., A smart local moving algorithm for large-scale modularity-based community detection. Eur. Phys. $J$. B, 2013, 86, 1-14.

23. Malik, V. S., Popkin, B. M., Bray, G. A., Despres, J. P., Willett, W. C. and Hu, F. B., Sugar-sweetened beverages and risk of metabolic syndrome and type-2 diabetes. Diabetes Care, 2010, 33, 2477-2483.

24. Holman, R. R., Paul, S. K., Bethel, M. A., Matthews, D. R. and Neil, H. A. W., 10-year follow-up of intensive glucose control in type-2 diabetes. N. Engl. J. Med., 2008, 359, 1577-1589.

ACKNOWLEDGEMENTS. This work was supported by the Scientific and Technological Innovation Research Funds for the Ministry of Science and Technology, People's Republic of China under Grant No. (ZLY2015147).

Received 9 December 2018; revised accepted 8 March 2019

doi: $10.18520 / \mathrm{cs} / \mathrm{v} 116 / \mathrm{i} 12 / 1965-1974$ 